\title{
The contribution of TMS to frontotemporal dementia variants
}

\begin{abstract}
Alberici A, Bonato C, Calabria M, Agosti C, Zanetti O, Miniussi C, Padovani A, Rossini PM, Borroni B. The contribution of TMS to frontotemporal dementia variants.

Acta Neurol Scand: DOI: 10.1111/j.1600-0404.2008.01017.x. (C) 2008 The Authors Journal compilation (c 2008 Blackwell Munksgaard.

Objective - Frontotemporal lobar degeneration (FTLD) includes different heterogeneous conditions mainly characterized by personality changes and cognitive deficits in language and executive functions; movement disorders have also been associated with FTLD. The present study aimed to measure the primary motor cortex (M1) inhibitory and facilitatory functions in patients affected by FTLD. Materials and methods - The study included 17 FTLD patients, 8 age-matched healthy controls and 8 Alzheimer's disease (AD) patients. Transcranial magnetic stimulation (TMS) was used to study intracortical inhibition (ICI) and facilitation (ICF) by using a double-pulse paradigm. Results - FTLD patients were comparable with controls and AD patients for ICI and ICF. Corticobasal degeneration (CBD) patients presented significant reduced inhibition at ISI3; moreover two out of seven CBD patients had only ipsilateral responses. Discussion - The present study reveals a selective impairment of M1 ICI inhibitory response in CBD, which may help in distinguishing among the FTLD clinical spectrum.
\end{abstract}

\author{
A. Alberici ${ }^{1}$, C. Bonato ${ }^{2}$ \\ M. Calabria ${ }^{3}$, C. Agosti ${ }^{1}$ \\ O. Zanetti ${ }^{4}$, C. Miniussi ${ }^{3,5}$, \\ A. Padovani', P. M. Rossini ${ }^{3,6}$, \\ B. Borroni ${ }^{1}$ \\ ${ }^{1}$ Department of Neurological Sciences, University of \\ Brescia, Italy; ${ }^{2}$ Casa di Cura Pederzoli, Peschiera (VR), \\ Italy; ${ }^{3}$ Neuropshysiology Unit, IRCCS S. Giovanni di \\ Dio-FBF, Brescia, Italy; ${ }^{4}$ Alzheimer Unit, IRCCS S. \\ Giovanni di Dio-FBF, Brescia, Italy; ${ }^{5}$ Department of \\ Biomedical Sciences and Biotechnologies, University of \\ Brescia, Italy; ${ }^{6} \mathrm{AFaR}$ - Department of Neuroscience, \\ Ospedale Isola Tiberina, Campus Biomedico, Rome, Italy \\ Key words: corticobasal degeneration; frontotemporal \\ lobar degeneration; Alzheimer disease; transcranial \\ magnetic stimulation; motor cortex, intracortical \\ inhibition-facilitation \\ Antonella Alberici, Department of Neurology, University \\ of Brescia, P.le Spedali Civili 1, 25100 Brescia, Italy \\ Tel.: +39-0303995603 \\ Fax: $+39-0303995027$ \\ e-mail: antoalberici@yahoo.it \\ Accepted for publication February 20, 2008
}

\section{Introduction}

Frontotemporal lobar degeneration (FTLD) is considered one of the most common forms of neurodegenerative dementia after Alzheimer disease (AD) (1). FTLD includes a wide spectrum of heterogeneous clinical and anatomical conditions, mainly characterized by social dysfunctions, personality changes along with cognitive impairment in language and executive functions (2). Language deficits are present in progressive non-fluent aphasia (PNFA), which occurs typically with dominant frontotemporal atrophy. If FTLD occurs with anomia and language comprehension impairment, it is named Semantic dementia (SD). When behavioral abnormalities are prominent, and usually associated with non-dominant temporal and frontal atrophy, the label of behavioral variant (bv-FTLD) is used $(3,4)$. In addition to social misconduct and cognitive deficits, FTLD is characterized by movement disorders that are variably represented by atypical extrapyramidal syndromes or motor neuron disease (MND). A common genetic and pathological overlap of FTLD with corticobasal degeneration (CBD) and progressive supranuclear palsy (PSP) suggested the inclusion of these relatively rare conditions under the same nosologic group (5). Several studies provided evidence that clinical and pathological features of FTLD and MND can occur, and therefore the term FTLD-MND is currently used to identify this specific entity $(6,7)$.

These different variants of FTLD tend to overlap as the disease progresses to other brain regions. Although with a different profile over time, motor deficits constitute an important disabling aspect, shared among all these FTLD conditions (8). Brain imaging has been used to characterize the different syndromes, allowing us to explore the involved neural systems, and helping us comprehend their pathogenetic mechanisms $(4,9-11)$. The question that remains open, however, is whether a single neural network underscoring FTLD or different entities might be discriminated in clinical practice.

Specific brain atrophy patterns in these different FTLD variants may underlie a distinctive and 
selective involvement of the primary motor cortex (M1).

The contribution of transcranial magnetic stimulation (TMS) has already demonstrated different neurophysiological patterns of $\mathrm{AD}$ as compared with FTLD $(12,13)$. It is known that an impaired cortex inhibition is considered an early feature in MND, and previous studies reported hyperexcitability of the motor cortex in atypical Parkinsonian syndromes (14-18). At present, however, intracortical inhibitory and facilitatory functions of motor cortex in FTLD and its variants still need to be completely clarified. We sought to explore this issue by adopting TMS technology, which allows effective, reliable and non-invasive methods for addressing motor cortex physiology, with the working hypothesis to provide a clinical tool for differentiating FTLD variants. Motor cortex hyperexcitability investigated by TMS could account for increased intrinsic excitability of cortical cells, or alternatively a reduced inhibition of cortical circuits. Inhibitory/facilitatory curves reflecting M1 intracortical excitability can be studied by using paired-pulse TMS (ppTMS) of conditioning and test stimuli, separated by a programmable time interval (19). We therefore aimed to assess the primary motor cortex inhibitory and facilitatory functions in a clinical series of FTLD specifically using ppTMS paradigm; comparisons were made with: (i) a group of $\mathrm{AD}$ patients and control subjects, and (ii) within the FTLD group, considering the different represented variants.

\section{Participants and methods}

Patients and participants

The study was approved by the local ethical committees and it included 17 FTLD patients, and 16 controls, including 8 AD patients and 8 healthy control subjects; informed consent was obtained from all subjects. Patients and control subjects were recruited from Department of Neurology, University of Brescia, and from Alzheimer Unit, IRCCS S Giovanni di Dio, Brescia. FTLD patients included seven patients with bv-FTD, three with PNFA and seven with CBD, according to published criteria $(3,5,20)$. In particular, bv-FTD was defined by character change and disorder of social conduct; PNFA was diagnosed when isolate disorder of expressive language was the first symptom with other aspects of cognition relatively well preserved; finally CBD when patients exhibited unilateral rigidity, apraxia, some of them having 'alien hand'; in these patients, the extrapyramidal syndrome developed first and was followed by cognitive changes and an additional inclusion criterion was the poor response to levo-DOPA treatment. AD diagnosis was based on criteria from National Institute of Neurological and Communicative Disorders and Stroke and the Alzheimer's Disease and Related Disorders Association (NINCDS-ADRDA) (21).

Routine laboratory tests including thyroid function, vitamin B12, serum folate and treponema serology were taken for all patients. All patients underwent extensive clinical and neurological examination. For each patient, a structural brain magnetic resonance imaging excluded major causes of cerebrovascular disease and white matter lesions. All FTLD patients underwent single photon emission computed tomography imaging for studying cerebral blood flow perfusion. Global cognitive function assessment was made according to a standardized battery, including the Mini-Mental State Examination (MMSE) (22). De Renzi Imitation Test was used to evaluate alien apraxia (23). Motor impairment was evaluated using the motor subscale of Unified Parkinson Disease Rating Scale (UPDRS) III (24). Instrumental Activities of Daily Living (IADL) and Basic Activities of Daily Living (BADL) were assessed as well (25, 26). Behavioral and psychiatric disturbances were evaluated by Frontal Behavioral Inventory (FBI) (27). Patients with mild or moderate cognitive decline (MMSE $\geq 18$ ) underwent extensive cognitive assessment, as described elsewhere (data not shown) (28). Patients with potentially confounding neurological and psychiatric disorders, clinically known hearing or vision impairment, a past history of alcohol abuse, psychosis, or major depression, were excluded from the study. All AD patients were on chronic administration of cholinesterase inhibitors. The use of other medications, including antidepressant, antipsychotic and benzodiazepine that could interfere with test performance at TMS was considered a further exclusion criterion. For TMS purposes, we excluded CBD with myoclonus at enrollment. Epilepsy-prone patients were excluded by anamnestic data and routine electroencephalogram recording.

\section{Paired-pulse paradigm}

The paired-pulse procedure was performed according to standardized methods (19). TMS was carried out by a Magstim Bistim magnetic stimulator and a circular coil having an inner diameter of $90 \mathrm{~mm}$ (Magstim Company Limited, Whitland, UK). A circular coil was used in order to minimize discomfort and optimize compliance from cognitively impaired patients. The coil had an inner diameter 
of $90 \mathrm{~mm}$; it was centered at the vertex and it was held tangentially to the scalp to excite preferentially the right hemisphere (current in the coil flowing clockwise) or the left hemisphere (current in the coil flowing anticlockwise). The $C z$ position was marked on the scalp to facilitate an exact repositioning of the coil center during the entire experiment and maintenance of the external edges on the two motor areas. The coil was stabilized and immobilized by means of a mechanical support. Once the coil was immobilized, the resting motor threshold was determined as the lowest stimulus intensity which produced in the first dorsal interosseus (FDI) muscle at least five motor evoked potential (MEP) of $50 \mu \mathrm{V}$ out of 10 consecutive stimuli (29). Amplitudes of the 'test' MEP were measured between the two major and stable peaks of opposite polarity and compared with those of a series of 'baseline', unconditioned (not preceded by the conditioning stimulus) MEP. Muscle twitches from either the left or the right hand triggered by TMS were recorded simultaneously from the FDI muscle, via $\mathrm{Ag} / \mathrm{AgCl}$ electrodes in a belly/tendon montage. Skin/electrode impedance was measured with the dedicated BrainVision module, and was confirmed to be $\leq 10 \mathrm{k} \Omega$. Signal recording was conducted using BrainAmp equipment (BrainProducts $\mathrm{GmbH}$, Munich, Germany) via $1-2000-\mathrm{Hz}$ filter setting and a poststimulus analysis time of $50 \mathrm{~ms}$ with a $5-\mathrm{KHz}$ sampling rate. The stimulus intensity for the first conditioning pulse (first) was set at $80 \%$ of the resting motor threshold. The (second) test pulse and the 'baseline' pulses were delivered suprathreshold with an intensity of $120 \%$ of the resting motor threshold. Interstimulus intervals (ISI) of 1,3 and $5 \mathrm{~ms}$ were selected to test short-interval intracortical inhibition (ICI). Meanwhile, ISI of 10 and $15 \mathrm{~ms}$ were used to test intracortical facilitation (ICF). Up to a maxi- mum of six trials with paired TMS were recorded for each ISI during complete muscle relaxation, whose order was also randomized. The size of conditioned responses (MEP amplitude) was expressed as a percentage of the unconditioned responses and plotted for each ISI. ICI and ICF were separately calculated for both: when the coil was oriented with the current flowing in clockwise as well as anticlockwise directions. Contralateral responses were considered those recorded in the left FDI with the coil oriented in order that the current flowed clockwise (preferential stimulation of the right hemisphere) and in the right FDI with the coil oriented in order that the current flowed anticlockwise (preferential stimulation of the left hemisphere). Ipsilateral responses were those recorded in the reverse order as described before. The amplitude of the conditioned MEP was expressed as the ratio of the 'baseline' MEP elicited by the test stimulus alone.

Data analysis

One-way ANOVA was performed for socio-demographic and clinical characteristics of patients and control subjects included in the study. One-way ANOVA was performed on ratios (conditioned/unconditioned response) in order to assess differences over groups (AD, FTLD and controls) and among clinical subgroups of FTLD. Analyses was separately performed for each ISI $(1,3,5,10$ and $15 \mathrm{~ms}$ ). Multiple comparisons were corrected by Bonferroni test. The level of significance was set at 0.05 .

\section{Results}

Main demographic and clinical features of patients and controls are reported in Table 1 . AD patients

Table 1 Main demographic and clinical features of controls, AD, FTLD and FTLD variants

\begin{tabular}{|c|c|c|c|c|c|c|c|c|c|}
\hline Diagnosis & $\begin{array}{c}\text { Gender, percent } \\
\text { female }\end{array}$ & Age, years & $\begin{array}{c}\text { Education, } \\
\text { years }\end{array}$ & $\begin{array}{l}\text { Disease duration, } \\
\text { months }\end{array}$ & MMSE & IADL & BADL & De Renzi & UPDRS III \\
\hline Controls, $n=8$ & 50 & $63.1(7.5)$ & $7.1(2.6)$ & - & $27.9(1.2)$ & 0 & 0 & - & - \\
\hline $\mathrm{AD}, n=8$ & 62.5 & $74.5(7.3)^{*}$ & $4.8(0.4)$ & $30.3(7.3)$ & $20.2(4.0)$ & $3.4(2.5)^{* *}$ & $1.1(1.5)$ & - & - \\
\hline FTLD, $n=17$ & 52.9 & $60.5(7.8)$ & $7.5(3)$ & $12.2(19.1)$ & $23.9(3.9)$ & $1.7(2.7)$ & $0.9(1.5)$ & 59.7 (12.7) & 16.62 (14.5) \\
\hline $\mathrm{CBD}, n=7$ & 57.1 & $58.7(9.4)$ & 7.7 (3.5) & $32.6(21.9)$ & $24.4(3.5)$ & $0.8(2.1)$ & $0.3(0.4)$ & 51.8 (7.0)\# & 30.5 (10.7)\# \\
\hline bv-FTD, $n=7$ & 42.9 & $63.4(6.7)$ & $7.4(3)$ & 31 (19.7) & $25.8(2.1)$ & $2.2(2.3)$ & $1.4(1.7)$ & $67.5(4.6)$ & $8.0(8.3)$ \\
\hline PNFA, $n=3$ & 66.7 & $58(1.6)$ & $7(1.4)$ & 24 & $18.3(3.3)^{\circ}$ & $2.6(3.7)$ & $1.3(1.9)$ & $59.0(4.5)$ & $9.0(6.4)$ \\
\hline
\end{tabular}

n, number; AD, Alzheimer disease; FTLD, frontotemporal lobar degeneration; CBD, corticobasal degeneration patients; bv-FTD, behavioral variant of FTLD; PNFA, progressive non-fluent aphasia; MMSE, Mini-Mental State Examination; BADL, Basic Activities of Daily Living; IADL, Instrumental Activities of Daily Living; De Renzi, De Renzi Imitation Test.

* Significantly different from FTLD and controls.

** Significantly different from controls.

\#Significantly different from bv-FTD and PNFA

- Significantly different from bv-FTD and CBD.

Standard deviations are given within brackets. 


\section{Alberici et al.}

were significantly aged than FTLD and controls. MMSE score differed significantly between controls and $\operatorname{AD}(P=0.0005)$, and FTD $(P=0.03)$ patients. Moreover, AD patients had lower ADL score as compared with controls $(P=0.02)$. Among FTLD subgroups, PNFA patients had significant lower MMSE score than CBD $(P=0.03)$, and bv-FTD $(P=0.008)$. CBD patients showed a lower score at De Renzi Imitation Test for right limb, and a higher score at UPDRS III.

\section{Resting motor threshold}

The resting motor threshold was as follows: $\mathrm{AD}$, mean $(\mathrm{M})=51.2[\mathrm{SD}]$ [7.8]; FTLD, $\mathrm{M}=50.9$ [11.1]; controls, $\mathrm{M}=54.2 \quad$ [6.5]; and within FTLD subgroup, in CBD, $\mathrm{M}=55$ [14.2]; bv-FTD, $\mathrm{M}=48.3$ [6.2]; PNFA, $\mathrm{M}=47.7$ [7.6]. No statistical differences were observed among FTLD, AD and controls and within FTLD variants.

\section{Contralateral responses}

No significant difference was found among $\mathrm{AD}$, FTLD and controls for ICI and ICF $(P>0.05)$, for responses recorded both when coil excited preferentially the right hemisphere (current in the coil flowing clockwise) or the left hemisphere (current in the coil flowing anticlockwise) (Fig. 1A).

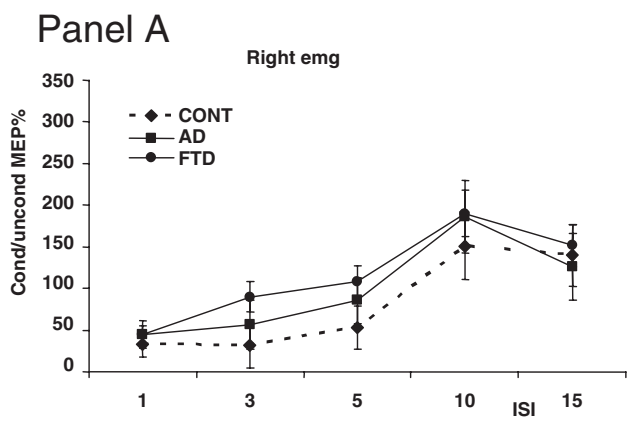

\section{Panel B}

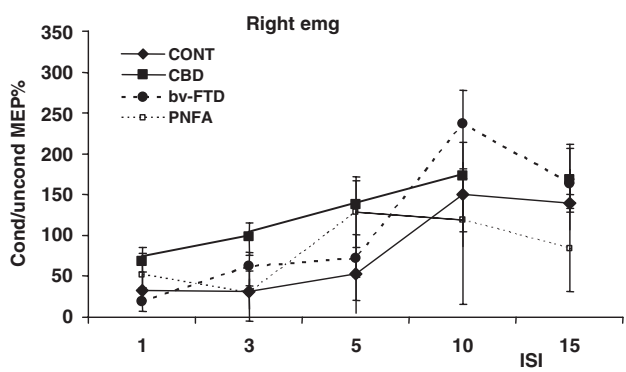

For the right hemisphere, considering only FTLD subgroups, we found a significant main effect of group $[F(3,21)=3.78, P=0.03)]$ only for ISI3. Post-hoc analysis revealed than CBD showed significant reduced inhibition (123\%) than controls $(29 \%, P=0.05)$ and bv-FTD (34\%, $P=0.02$ ) (Fig. 1B).

For the left hemisphere, considering only FTLD subgroups, we found a significant main effect of group $[F(3,21)=3.21, P=0.04)]$ only for ISI3. Post-hoc analysis revealed that CBD showed significantly reduced inhibition $(98 \%)$ than controls $(31 \%, P=0.05)$ (Fig. 1B).

Ipsilateral responses

No significant difference was found among AD, FTLD and controls for ICI and ICF $(P>0.05)$, for both hemispheres. For the right hemisphere, considering only FTLD subgroups, we found a significant main effect of group $[F(3,21)=4.65$, $P=0.01$ )] only for ISI3. Post-hoc analysis revealed that $\mathrm{CBD}$ showed significantly reduced inhibition $(93 \%)$ than controls $(31 \%, P=0.03)$ and PNFA $(15 \%, P=0.01)$. No significant difference was found for the left hemisphere among FTLD subgroups.

Two out of seven CBD patients showed only ipsilateral responses to stimulation and no contralateral MEP (Fig. 2).
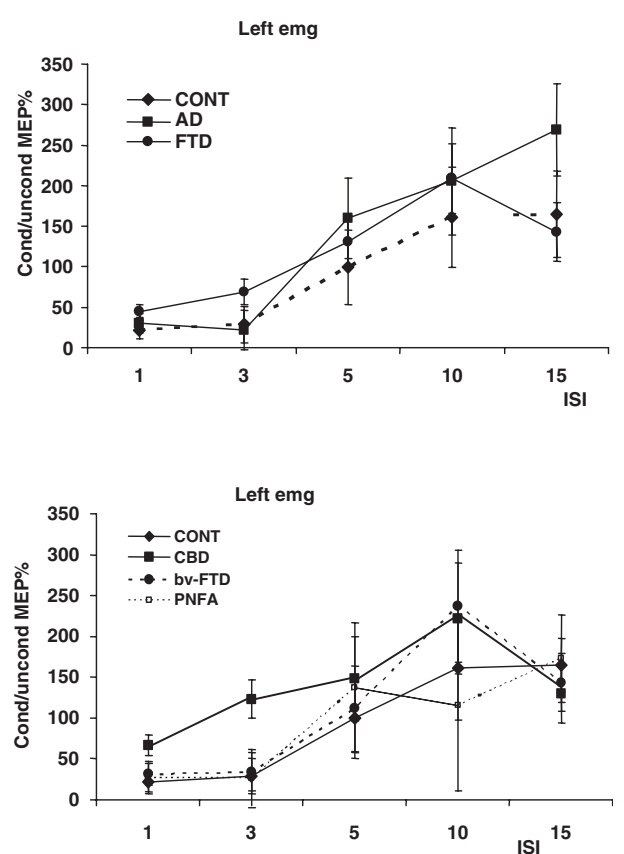

Figure 1. (A) Contralateral responses of primary motor cortex (M1) inhibitory/facilitatory curves in frontotemporal lobar degeneration (FTLD), Alzheimer disease and healthy controls. (B) Contralateral responses of M1 inhibitory/facilitatory curves in corticobasal degeneration patients, behavioral variant of FTLD, progressive non-fluent aphasia and healthy controls. ISI indicates interstimulus intervals. 

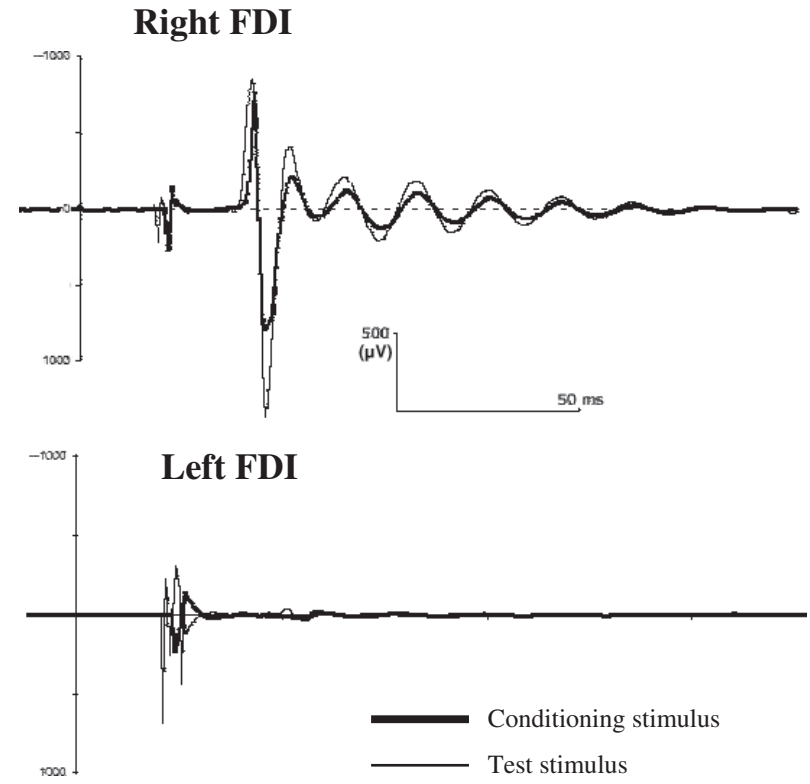

Figure 2. Coil oriented in order that the current flowed clockwise with preferential stimulation of the right hemisphere: representative ipsilateral motor-evoked potential in a corticobasal degeneration patient.

\section{Discussion}

FTLD is currently differentiated in different variants, and these clinical distinctions have specific imaging and pathological correlations. The mismatch, however, between clinical pictures and pathology makes the present classification largely unsatisfactory in clinical practice (30).

In this study, we approached these issues, employing the ppTMS paradigm, and applying it to a group of FTLD patients. In the first instance comparisons were made among FTLD, AD and a control group, and second within the variously represented FTLD variants, with the attempt to evaluate the inhibitory (ICI) and facilitatory (ICF) functions of motor cortex. ICI and ICF result from the balance between intracortical inhibitory circuits mediated through gamma-aminobutyric acid (GABA)ergic transmission, and excitatory activity mainly sustained by glutamate modulation (31).

In the present study, we showed that $\mathrm{AD}$ and FTLD preserve normal inhibitory and facilitatory functions of motor cortex as compared with the controls. Increased motor cortex excitability has been demonstrated in AD by different studies, but the underlying mechanisms are still to be completely defined $(13,32)$. In our AD patients, normal ICI functions reflected a still intact GABAergic inhibitory tone, in early stages of the disease. These data suggest the hypothesis that the previously observed motor cortex hyperexcitabil- ity may reflect impairment in cholinergic activity, but at the same time may be the result of an abnormal N-methyl-D-aspartic acid transmission, thus providing an additional rationale to treatments with cholinesterase inhibitors and memantine for AD at disease onset (33).

When considered as a whole, our FTLD patients exhibited normal patterns of ICI and ICF, similar to what was found in other experimental settings $(12,13)$. Notwithstanding, within the represented FTLD variants, CBD patients were selectively impaired in inhibitory functions compared with the other subgroups. This suggests that GABAergic circuits might be defective in these patients, but relatively preserved in bv-FTD and PNFA. CBD was known to be characterized by an abnormal cortical excitability, that has been attributed to a reduced cortical and transcallosal inhibition, and to an enlargement of cortical map for hand representation; ispilateral motor responses were also previously observed $(16,18)$. In our experimental settings, the use of the circular coil gave a further demonstration of this phenomenon, that may be related to the peculiar clinical characteristics of CBD, particularly in transcallosal transmission $(34,35)$. The level of motor impairment as documented by worse UPDRS score and apraxia deficits by De Renzi Imitation Test in CBD may account for such differences in ppTMS paradigm as compared with bv-FTD and PNFA. We acknowledge that these results have some limitations: the data should be replicated in a larger sample size, including PSP and FTLD-MND patients, but we can argue that TMS may help clinicians in the challenging issue of clinical diagnosis within FTLD variants. Eventually, the observed deficit in ICI functions of CBD motor cortex may provide a possible explanation of one of the core features of this disease, such as cortical myoclonus. Indeed, the GABA neurotransmission involvement further supports a potential framework for an evidence-based treatment with GABAergic medications in CBD.

\section{Acknowledgements}

The authors are grateful to the patients and their families for their generous collaboration. They thank Mrs Mary Gilman for kindly editing the manuscript.

\section{References}

1. Snowden JS, Neary D, Mann DM. Frontotemporal dementia. Br J Psychiatry 2002;180:140-3.

2. Hodges JR, Davies RR, Хuereb JH et al. Clinicopathological correlates in frontotemporal dementia. Ann Neurol 2004;56:399-406. 


\section{Alberici et al.}

3. Neary D, Snowden JS, Gustafson L et al. Frontotemporal lobar degeneration: a consensus on clinical diagnostic criteria. Neurology 1998;51:1546-54.

4. Rosen HJ, Allison SC, Schauer GF et al. Neuroanatomical correlates of behavioural disorders in dementia. Brain 2005; 128:2612-25.

5. Mckhann GM, Albert MS, Grossman M et al. Clinical and pathological diagnosis of frontotemporal dementia: report of the Work Group on Frontotemporal Dementia and Pick's Disease. Arch Neurol 2001;11:1803-9.

6. Caselli RJ, Windebank AJ, Petersen RC et al. Rapidly progressive aphasic dementia and motor neuron disease. Ann Neurol 1993;33:200-7.

7. Lomen-Hoerth C, Anderson T, Miller B. The overlap of amyotrophic lateral sclerosis and frontotemporal dementia. Neurology 2002;59:1077-9.

8. Kertesz A, Mcmonagle P, Blair M et al. The evolution and pathology of frontotemporal dementia. Brain 2005; 128:1996-2005.

9. Miller BL, Seeley WW, Mychack P et al. Neuroanatomy of the self: evidence from patients with frontotemporal dementia. Neurolog 2001;57:817-21.

10. Franceschi M, Anchisi D, Pelati $\mathrm{O}$ et al. Glucose metabolism and serotonin receptors in the frontotemporal lobe degeneration. Ann Neurol 2005;57:216-25.

11. Padovani A, Borroni B, Brambati SM et al. Diffusion tensor imaging and voxel based morphometry study in early progressive supranuclear palsy. J Neurol Neurosurg Psychiatry 2006;77:457-63.

12. Pierantozzi M, Panella M, Palmieri MG et al. Different TMS patterns of intracortical inhibition in early onset Alzheimer dementia and frontotemporal dementia. Clin Neurophysiol 2004;115:2410-8.

13. Di Lazzaro V, Pilato F, Dileone $\mathrm{M}$ et al. In vivo cholinergic circuit evalutaion in frontotemporal and Alzheimer dementias. Neurology 2006;66:1111-13.

14. Ziemann U, Winter $M$, Reimers $C$ et al. Impaired motor cortex inhibition in patients with ALS: evidence from paired trancranial magnetic stimulation. Neurology 1998;51:1771-2.

15. Vucic S, Kiernan MC. Novel threshold tracking techniques suggest that cortical hyperexcitability is an early feature of motor neuron disease. Brain 2006;129:2436-46.

16. Hanajima R, Ugawa $\mathrm{Y}$, Terao $\mathrm{Y}$ et al. Ipsilateral corticocortical inhibition of the motor cortex in various neurological disorders. J Neurological Sci 1995;140:109-16.

17. Kunn AA, Grosse P, Holtz K et al. Patterns of abnormal motor cortex excitability in atypical parkinsonian syndromes. Clin Neurophysiol 2004;115:1786-95.

18. Valls-Solè J, Tolosa E, Marti MJ et al. Examination of motor output pathways in patients with corticobasal ganglionic degeneration using transcranial magnetic stimulation. Brain 2001;124:1131-7.

19. Kujirai T, Caramia MD, Rothwell JC et al. Corticocortical inhibition in human motor cortex. J Physiol 1993;471:501519
20. LANG AE. Cortical-basal ganglionic degeneration. In: CALNE DB, ed. Neurodegenerative disease. Saunders: Philadelphia, 1994;877-94.

21. McKhann G, Drachman D, Folstein $M$ et al. Clinical diagnosis of Alzheimer's disease: report of the NINCDSADRDA Work Group under the auspices of Department of Health and Human Services Task Force on Alzheimer's disease. Neurology 1984;34:939-44.

22. Folstein MF, Folstein SE, Mchugh PR. "Mini-Mental State": a practical method for grading the cognitive state of patients for the clinician. J Psychiatr Res 1975;12:189-98.

23. De Renzi E, Motti F, Nichelli P. Imitating gestures. A quantitative approach to ideomotor apraxia. Arch Neurol 1980;37:6-10.

24. Louis ED, Lynch T, Marder K, Fahn S. Reliability of patient completion of the historical section of the United Parkinson's Disease Rating Scale. Mov Disord 1996; 11:185-92.

25. Lawton MP, Broody EM. Assessment of older people: selfmaintaining and instrumental activities of daily living. Gerontologist 1969;9:179-86.

26. Sheirh K, Smith DS, Meade TW et al. Repeatibility and validity of a modified Activities of Daily Living (ADL) Index in studies of chronic disability. Int Rehabil Med 1979;1:51-8.

27. Kertesz A, Nadkarni N, Davidson W, Thomas AW. The Frontal Behavioral Inventory in the differential diagnosis of frontotemporal dementia. J Int Neuropsychol Soc 2000;6:460-8.

28. Cotelli M, Borroni B, Manenti $\mathrm{R}$ et al. Action and object naming in frontotemporal dementia, progressive supranuclear palsy, and corticobasal degeneration. Neuropsychology 2006;20:558-65.

29. Rossini PM, Baker AT, Berardelli A et al. Non invasive electrical and magnetic stimulation of the brain, spinal cord and roots: basic priciples and procedures for routine clinical application. Electroencephalogr Clin Neurophysiol 1994;91:79-92.

30. Forman MS, Farmer J, Johnson JK et al. Frontotemporal dementia: clinicopathological correlations. Ann Neurol 2006;59:952-62.

31. Ziemann U. TMS and drugs. Clin Neurophysiol 2004; 115:1717-29.

32. Ferreri $\mathrm{F}$, Pauri F, Pasqualetti $\mathrm{P}$ et al. Motor cortex excitability in Alzheimer's disease: a transcranial magnetic stimulation study. Ann Neurol 2003;53:102-8.

33. Rabins PV, LyKetsos CG. Cholinesterase inhibitors and memantine have a role in the treatment of Alzheimer's disease. Nat Clin Pract Neurol 2006;2:578-9.

34. Окuma $\mathrm{Y}$, Urabe $\mathrm{T}$, Mochizuki $\mathrm{H}$ et al. Asymmetric cortico-cortical inhibition in patients with progressive limb-kinetic apraxia. Acta Neurol Scand 2000;102:244-8.

35. Trompetto C, Buccolieri A, Marchese R, Marinelli L, Michelozzi G, Abbruzzese G. Impairment of transcallosal inhibition in patients with corticobasal degeneration. Clin Neurophysiol 2003;114:2181-7. 